\title{
BSM MODEL FOR THE GENERALIZED ML-PAYOFF
}

\author{
S.J. Ghevariya, D.R. Thakkar \\ Department of Mathematics, Sardar Patel University \\ Vallabh Vidyanagar, India \\ sj_ghevariya@spuvvn.edu, dharathakkar29796@gmail.com
}

Received: 2 July 2019; Accepted: 26 November 2019

\begin{abstract}
The Black-Scholes-Merton (BSM) model for the modified log payoff (ML-Payoff) function has been derived by Denania and Ghevariya. This paper focuses on the derivation of the BSM model for the Generalized ML-Payoff function. The objective of this paper is the derivation of the BSM model which is quite close to the celebrated BSM model for the plain vanilla payoff function. Note that Paul Wilmott's BSM model of log payoff has become a special case of this model.
\end{abstract}

MSC 2010: $91 B 25,91 G 20$

Keywords: BSM model, Plain vanilla payoff, ML-Payoff, Generalized ML-Payoff

\section{Introduction}

One of the most important financial derivatives are options. Options can be used to hedge assets and portfolios to control risk due to volatility in asset prices. So the natural question is how to value an option? In the early 1970s, Fischer Black, Myron Scholes and Robert Merton achieved a major breakthrough in this direction. They derived a mathematical model known as the Black-Scholes-Merton (BSM) model for valuing options for the plain vanilla payoff. The BSM model for the plain vanilla payoff has been used by millions of people worldwide in financial markets. After that, BSM models for pricing options have become one of the major areas in financial study over the last five decades. Hence several other BSM models were derived for various linear and non linear payoffs [1]. The BSM model for the modified log payoff (ML-Payoff) has been derived through different approaches [2-5]. This is a modified version of Paul Wilmott's BSM model of log payoff [6, p. 149]. In this paper, we discuss the closed form solution of the BSM model of the European style on an asset paying no dividend. It seems difficult to derive closed form solutions of these models for American style options [7]. In section 2, we shall derive the BSM model for the Generalized ML-Payoff. In section 3, we discuss some special cases from the BSM model for the Generalized ML-Payoff. In section 4, the comparisons of the BSM model for ML-Payoffs and plain vanilla payoffs have been provided with the use of graphs. First, we define the Generalized ML-Payoff. 
Definition 1 The Generalized ML-Payoff for the European call option is defined as

$$
C^{E}(S, T)= \begin{cases}P\left(S_{T}\right) \ln \left(\frac{S_{T}}{K}\right), & S_{T} \geq K, \\ 0, & \text { otherwise }\end{cases}
$$

and the put option is defined as

$$
P^{E}(S, T)= \begin{cases}P\left(S_{T}\right) \ln \left(\frac{K}{S_{T}}\right), & S_{T} \leq K \\ 0, & \text { otherwise }\end{cases}
$$

where $P: \mathbb{R}^{+} \rightarrow \mathbb{R}^{+}$defined by $P(x)=\sum_{l=0}^{n} a_{l} x^{l}, n \in \mathbb{N} \cup\{0\}, a_{l} \in \mathbb{R}^{+}, K$ is the striking price, and $S_{T}$ is the value of underlying asset (e.g., financial or commodity) at expiration time $T$.

\section{BSM model for the Generalized ML-Payoff}

In this section, the derivation of the BSM model for the Generalized ML-Payoff for a call option has been discussed and the put option has been stated. Further, the BSM models for plain vanilla options have been stated.

THEOREM 2.1 The BSM model for the Generalized ML-Payoff for a call option is

$$
\begin{aligned}
C^{E}= & \sum_{l=0}^{n} a_{l} S_{t}^{l} e^{(l-1)\left(l+\frac{1}{2} r \sigma^{2}\right)(T-t)}\left[\zeta\left(d_{l}\right) \sigma \sqrt{T-t}\right. \\
& \left.+\left(\ln \left(\frac{S_{t}}{K}\right)+\left(r+\frac{1}{2}(2 l-1) \sigma^{2}\right)(T-t)\right) N\left(d_{l}\right)\right],
\end{aligned}
$$

where

$d_{l}=\frac{\ln \left(\frac{S_{t}}{K}\right)+\left(r+\frac{1}{2}(2 l-1) \sigma^{2}\right)(T-t)}{\sigma \sqrt{T-t}}, \sqrt{2 \pi} \zeta\left(d_{l}\right)=e^{-\frac{d_{l}^{2}}{2}}$ and $N(\cdot)$ is the cdf of standard normal random variable.

PROOF The BSM differential equation with the boundary conditions for the European call option $C^{E}$ is

$$
\frac{\partial C^{E}}{\partial t}+\frac{1}{2} \sigma^{2} S_{t}^{2} \frac{\partial^{2} C^{E}}{\partial S_{t}^{2}}+r S_{t} \frac{\partial C^{E}}{\partial S_{t}}-r C^{E}=0
$$

with $C^{E}(0, t)=0, C^{E}\left(S_{t}, t\right) \rightarrow S_{t}$ whenever $S_{t} \rightarrow \infty$ and $C^{E}(S, T)=\max \left\{P\left(S_{T}\right) \ln \left(\frac{S_{T}}{K}\right), 0\right\}$, where $r$ is the risk free interest rate and $\sigma$ is the volatility. By taking $S_{t}=K e^{x}$, 
$y=\frac{1}{2} \sigma^{2}(T-t), C^{E}=K g(x, y)$, Equation (4) becomes

$$
\frac{\partial g}{\partial y}=\frac{\partial^{2} g}{\partial x^{2}}+(p-1) \frac{\partial g}{\partial x}-p g
$$

where $p=\frac{r}{\frac{1}{2} \sigma^{2}}$. Again, by taking

$$
f(x, y)=e^{\frac{1}{2}(p-1) x+\frac{1}{4}(p+1)^{2} y} g(x, y),
$$

Equation (5) becomes

$$
\frac{\partial f}{\partial y}=\frac{\partial^{2} f}{\partial x^{2}} \quad(y>0, x \in \mathbb{R})
$$

Note that we have $C^{E}(S, T)=\max \left\{P\left(S_{T}\right) \ln \left(\frac{S_{T}}{K}\right), 0\right\}$ which gives

$$
K f(x, 0)= \begin{cases}x e^{\frac{1}{2}(p-1) x} P\left(K e^{x}\right), & x \geq 0 \\ 0, & x<0 .\end{cases}
$$

Note that Equation (5) is a heat equation and hence the solution is

$$
f(x, y)=\frac{1}{2 \sqrt{\pi y}} \int_{-\infty}^{\infty} f(s, 0) e^{-\frac{(s-x)^{2}}{4 y}} d s,
$$

where $f(x, 0)$ is given by Equation (8). Combining Equations (8) and (9), we get

$$
K f(x, y)=e^{\frac{1}{2}(p-1) x}\left[I_{1}+I_{2}\right],
$$

where

$$
I_{1}=x \sum_{l=0}^{n} a_{l} K^{l} e^{l x} \frac{1}{\sqrt{2 \pi}} \int_{\frac{-x}{\sqrt{2 y}}}^{\infty} e^{-\frac{1}{2}\left(s^{2}-\sqrt{2 y}(2 l+p-1) s\right)} d s
$$

and

$$
I_{2}=\sqrt{2 y} \sum_{l=0}^{n} a_{l} K^{l} e^{l x} \frac{1}{\sqrt{2 \pi}} \int_{\frac{-x}{\sqrt{2 y}}}^{\infty} s e^{-\frac{1}{2}\left(s^{2}-\sqrt{2 y}(2 l+p-1) s\right)} d s .
$$

Equations (11) and (12) can be written as

$$
I_{1}=x \sum_{l=0}^{n} a_{l} K^{l} e^{l x+\frac{1}{4}(p+2 l-1)^{2} y} N\left(d_{l}\right)
$$

and

$$
I_{2}=\sum_{l=0}^{n} a_{l} K^{l} e^{l x+\frac{1}{4}(p+2 l-1)^{2} y}\left[\zeta\left(d_{l}\right) \sqrt{2 y}+(p+2 l-1) y N\left(d_{l}\right)\right]
$$


where

$$
d_{l}=\frac{x+(p+2 l-1) y}{\sqrt{2 y}}, \quad \zeta\left(d_{l}\right)=\frac{1}{\sqrt{2 \pi}} e^{-\frac{d_{l}^{2}}{2}} \quad \text { and } \quad N(x)=\int_{-\infty}^{x} \zeta(x) d x .
$$

Substituting values of Equations (13) and (14) into Equation (10), we get

$$
K f(x, y)=\sum_{l=0}^{n} a_{l} K^{l} e^{\frac{1}{2}(p+2 l-1) x+\frac{1}{4}(p+2 l-1)^{2} y}\left[\zeta\left(d_{l}\right) \sqrt{2 y}+(x+(p+2 l-1) y) N\left(d_{l}\right)\right]
$$

From Equation (6), we get

$$
K g(x, t)=\sum_{l=0}^{n} a_{l} K^{l} e^{l x+(l-1)(p+l) y}\left[\zeta\left(d_{l}\right) \sqrt{2 y}+(x+(p+2 l-1) y) N\left(d_{l}\right)\right]
$$

Substituting $S_{t}=K e^{x}, y=\frac{1}{2} \sigma^{2}(T-t)$ and $C^{E}=K g(x, y)$ in Equation (15), we have

$$
\begin{aligned}
C^{E}= & \sum_{l=0}^{n} a_{l} S_{t}^{l} e^{(l-1)\left(r+\frac{1}{2} l \sigma^{2}\right)(T-t)}\left[\zeta\left(d_{l}\right) \sigma \sqrt{T-t}\right. \\
& \left.+\left(\ln \left(\frac{S_{t}}{K}\right)+\left(r+\frac{1}{2}(2 l-1) \sigma^{2}\right)(T-t)\right) N\left(d_{l}\right)\right],
\end{aligned}
$$

where $d_{l}, \zeta\left(d_{l}\right)$ and $N(\cdot)$ are given in Equation (3). Equation (16) is the BSM model for the Generalized ML-Payoff for call option.

THEOREM 2.2 The BSM model for the Generalized ML-Payoff for put option is

$$
\begin{aligned}
P^{E}= & \sum_{l=0}^{n} a_{l} S_{t}^{l} e^{(l-1)\left(r+\frac{1}{2} l \sigma^{2}\right)(T-t)}\left[\zeta\left(d_{l}\right) \sigma \sqrt{T-t}\right. \\
& \left.-\left(\ln \left(\frac{S_{t}}{K}\right)+\left(r+\frac{1}{2}(2 l-1) \sigma^{2}\right)(T-t)\right) N\left(-d_{l}\right)\right],
\end{aligned}
$$

where $d_{l}, \eta\left(d_{l}\right)$ and $N(\cdot)$ are given in Equation (3).

THEOREM 2.3 [1] The BSM models of European options for plain vanilla payoffs $C_{1}^{E}(S, T)=\max \left\{S_{T}-K, 0\right\}$ and $P_{1}^{E}(S, T)=\max \left\{K-S_{T}, 0\right\}$ are given by

$$
C_{1}^{E}=S_{t} N\left(d_{1}\right)-K e^{-r(T-t)} N\left(d_{2}\right)
$$

and

$$
P_{1}^{E}=K e^{-r(T-t)} N\left(-d_{2}\right)-S_{t} N\left(-d_{1}\right),
$$

where $d_{1}, d_{2}$ and $N(\cdot)$ are given in Equation (3). 


\section{Corollaries}

In this section, we shall derive some special cases from the BSM model for the Generalized ML-Payoff discussed above. Note that $d_{1}$ in the following equations are as per Equation (3).

CASE-I: The BSM model for ML-Payoff [2].

Taking $p(x)=x$ in Equations (1) and (2), we get ML-Payoffs $C_{2}^{E}(S, T)=$ $=\max \left\{S_{T} \ln \left(\frac{S_{T}}{K}\right), 0\right\}$ and $P_{2}^{E}(S, T)=\max \left\{S_{T} \ln \left(\frac{K}{S_{T}}\right), 0\right\}$. Then the corresponding formulas are

$$
\begin{aligned}
& C_{2}^{E}=S\left[\zeta\left(d_{1}\right) \sigma \sqrt{T-t}\left(\ln \left(\frac{S_{t}}{K}\right)+\left(r+\frac{1}{2} \sigma^{2}\right)(T-t)\right) N\left(d_{1}\right)\right], \\
& P_{2}^{E}=S\left[\zeta\left(d_{1}\right) \sigma \sqrt{T-t}\left(\ln \left(\frac{S_{t}}{K}\right)-\left(r+\frac{1}{2} \sigma^{2}\right)(T-t)\right) N\left(-d_{1}\right)\right] .
\end{aligned}
$$

CASE-II: The BSM model for log payoff [6].

Taking $p(x)=1$ in Equations (1) and (2), we get the $\log$ payoffs $C_{3}^{E}(S, T)=$ $=\max \left\{\ln \left(\frac{S_{T}}{K}\right), 0\right\}$ and $P_{3}^{E}(S, T)=\max \left\{\ln \left(\frac{K}{S_{T}}\right), 0\right\}$. Then the corresponding formulas are

$$
\begin{aligned}
& C_{3}^{E}=e^{-r(T-t)}\left[\zeta\left(d_{1}\right) \sigma \sqrt{T-t}\left(\ln \left(\frac{S_{t}}{K}\right)+\left(r-\frac{1}{2} \sigma^{2}\right)(T-t)\right) N\left(d_{1}\right)\right], \\
& P_{3}^{E}=e^{-r(T-t)}\left[\zeta\left(d_{1}\right) \sigma \sqrt{T-t}\left(\ln \left(\frac{S_{t}}{K}\right)-\left(r-\frac{1}{2} \sigma^{2}\right)(T-t)\right) N\left(-d_{1}\right)\right] .
\end{aligned}
$$

\section{Comparison}

The BSM model for the plain vanilla payoff is the most popular in the financial markets. So the comparison of the BSM model for the plain vanilla payoff function with the BSM model for other payoff function will be worthwhile [8]. Therefore, in this section, we compare Paul Wilmott's BSM models for log payoff functions and BSM models for ML-Payoff functions with the BSM models for the plain vanilla payoff functions. Throughout this section, we fix the current asset price $S_{0}=100$, the maturity time $T=0.5$ and the risk free interest rate $r=0.08$; using these, we draw the graphs of call and put option values verses volatility $(\sigma)$ of the underlying asset and the striking price $(K)$. 


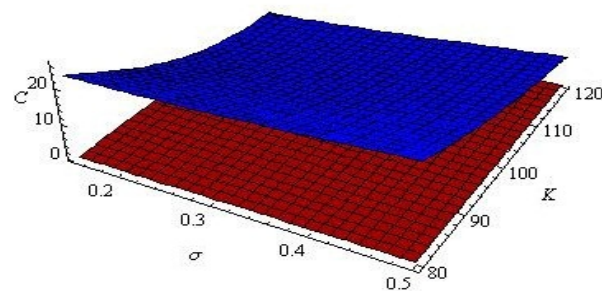

Fig. 1. Call option values of plain vanilla $\left(C_{1}^{E}\right)$ and $\log \left(C_{3}^{E}\right)$

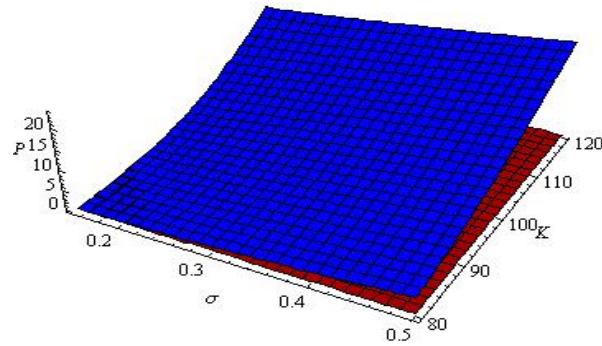

Fig. 3. Put option values of plain vanilla $\left(P_{1}^{E}\right)$ and $\log \left(P_{3}^{E}\right)$

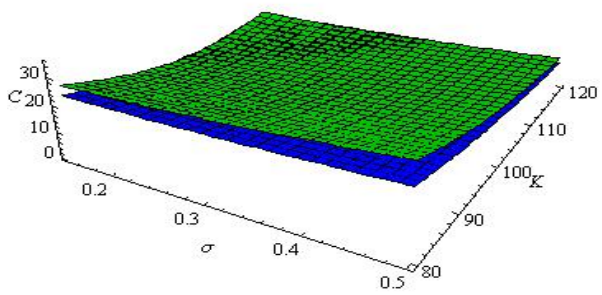

Fig. 2. Call option values of plain vanilla $\left(C_{1}^{E}\right)$ and modified $\log \left(C_{2}^{E}\right)$

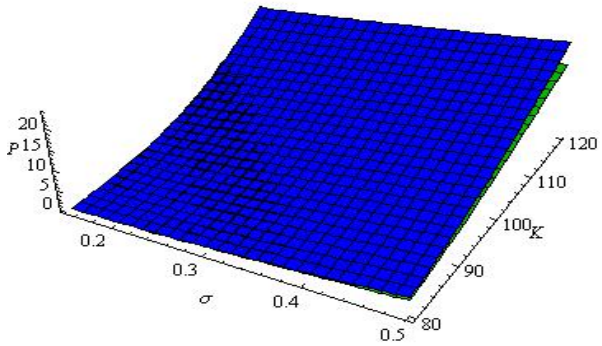

Fig. 4. Put option values of plain vanilla $\left(P_{1}^{E}\right)$ and modified $\log \left(P_{2}^{E}\right)$

\section{Conclusions}

From the Figures 1-4, it can be seen that the BSM model for the ML-Payoff function is quite close to the BSM model for the plain vanilla payoff function rather than the BSM model for the log payoff function. Further, the important conclusion is that the call option values of modified log are higher than plain vanilla. So the writer is more beneficial in terms of premium to enter into a modified log call option compared to plain vanilla call option. Similarly, the holder is more beneficial to enter into a put option using the same. This paper contributes to the derivation of the BSM model for the Generalized ML-Payoff function which might be very close to plain vanilla by taking an appropriate polynomial in the Generalized ML-Payoff.

\section{References}

[1] Haug, E.G. (2007). The Complete Guide to Option Pricing Formulas. 2nd ed., McGraw-Hill.

[2] Dedania, H.V., \& Ghevariya, S.J. (2013). Option pricing formula for modified log-Payoff function. International Journal of Mathematics and Soft Computing, 3(2), 129-140.

[3] Ghevariya, S.J. (2018). BSM European put option pricing formula for ML-Payoff function with Mellin transform. Int. Jr. of Mathematics and Its Applications, 6(2), 33-36. 
[4] Ghevariya, S.J. (2019). An improved Mellin tranform approach to BSM formula of ML-Payoff function. Journal of Interdisciplinary Mathematics. Taylor and Francis Group (accepted).

[5] Ghevariya, S.J. (2019). BSM model for ML-Payoff function through PDTM. Asian-European Journal of Mathematics. World Scientific Publisher, To apear.

[6] Wilmott, P. (2006). Paul Wilmott on Quantitative Finance. 2nd ed., John Wiley \& Sons.

[7] Wilmott, P., Howison, S., \& Dewynne, J. (2002). Mathematics of Financial Derivatives. Cambridge University Press.

[8] Dedania, H.V., \& Ghevariya, S.J. (2017). Graphical interpretation of various BSM formulas. Global Journal of Pure and Applied Mathematics, 13(9), 6107-6112. 